\title{
Use Stock Market Data to assist investors on getting more accurate transaction decision
}

\author{
A.V.L. Chandima \\ Department of Information Technology \\ Advanced Technological Institute \\ Sri Lanka Institute of Advanced Technological Education \\ Sri Lanka \\ DOI: 10.29322/IJSRP.11.01.2021.p10993 \\ http://dx.doi.org/10.29322/IJSRP.11.01.2021.p10993
}

\begin{abstract}
Colombo Stock Exchange (CSE) is one of the best performing stock exchanges in South Asia. Presently lots of investors (i.e. people who buy or sell stocks) connected with the CSE. Generally stock market domain is a dynamic and unpredictable environment. However, stock trading (buy / sell) process doesn't exist without prediction. In the current stock trading process investors predict the following day stock price solely based on the stock broker's assistant. Stock brokers are the one who directly connects with the stock trading process regularly. Therefore, they have massive experience and ongoing knowledge, so they can use this to assist the investors. But current stock trading process in CSE, stock brokers do not have much time to completely disclose their knowledge and assist investors uniformly. Also, in some situations stock brokers are not aware of several patterns based on the previous data, but they are more valuable for the investors to do their stock trading process. So, most investors are unable to win their stock trading process and gain profit.
\end{abstract}

\section{Key word}

Stock trading, Stock brokers, investors, Stock exchange, Stock market, Stock market prediction, Technical analysis, Technical indicators, Share trading Colombo stock exchange, Share trading

\section{Introduction}

Stock trading means to buying and selling stocks in financial terms. Stock trading collect vast amounts of temporal (i.e., time associated) data such as trade date, Closing stock price, high stock price, low stock price, number of shares, etc. So, within a year we can collect around $5500 \mathrm{~KB}$ of data and this amount is expected to continue to grow considerably in the future. Data extraction leads to discover valuable knowledge. Using this knowledge investors can accurately disclose the intelligent investment decisions. But if this much of data wealth are available, can investors fully capitalize on the exactions of data? Data mining [1], the science and technology of exploring data in order to discover previously unknown patterns, is a part of the overall process of knowledge discovery in database (KDD). In today's computer-driven world, these databases contain massive quantities of information. The accessibility and abundance of this 
information makes data mining a matter of considerable importance and necessity.

Data mining, the extraction of hidden predictive information from large databases, is a powerful new technology with great potential to help business or market related companies to focus on the most important information in their data warehouses. Data mining tools predict future trends and behaviors, allowing businesses to make proactive, knowledgedriven decisions. The automated, prospective analyses offered by data mining move beyond the analyses of past events provided by retrospective tools typical of decision support systems. Data mining techniques are the result of a long process of research and product development. This evolution began when business data was first stored on computers, continued with improvements in data access, and more recently, generated technologies that allow users to navigate through their data in real time. The ultimate goal of data mining is prediction. Predictive data mining is the most common type of data mining and one that has the most direct business applications. The term Predictive Data Mining is usually applied to identify data mining projects with the goal to identify a statistical or neural network model or set of models that can be used to predict some response of interest. Financial market analysis mostly used supervised machine learning techniques.

National economies are strongly linked and heavily influenced of the performance of their Stock Markets [2]. So, the characteristic that all Stock Markets have in common is the uncertainty, which is related with their short and long-term future state. This feature is undesirable for the investor but it is also unavoidable whenever the Stock Market is selected as the investment tool. The best that one can do is to try to reduce this uncertainty. Stock Market Prediction [2] (or Forecasting) using the data mining is one of the instruments in this process. By analyzing the literature there are three Stock Market Prediction methods can be introduced. These methods are Fundamental analysis, Technical analysis and Technological analysis.

Fundamental analysis concerns the tenets of the company's foundation theory to the selection of individual stocks. This type of analysis is not possible to fit in the objectives of the proposed system. The reason for this is that the data it uses in order to determine the stock value does not change on daily basis because it uses the intrinsic value of the asset. Therefore, fundamental analysis is helpful for predicting the market only in a long-term basis. Technological analysis is another method used in stock trading. In this method stock market prediction moves into the technological realm. The most prominent techniques involved are Artificial Neural Network and Genetic Algorithms. Technical analysis seeks to determine the future price of a stock based solely on the (potential) trends of the past stock prices. Past stock prices are analyzed using the tool called technical indicators and correlated charts. These charts can be used to discover numerous patterns in stock trading process and predict the future stock trading direction.

\section{Methodology}

Technical analysis is a stock market prediction method based on the analysis of changes in a stock's price and volume of trading. Technical stock analysts construct a variety of charts plotting stock-price changes. Based on these charts, investors predict future price movement. Technical analysts or chartists are not concerned with any of the company's fundamentals. 
They seek to determine the future price of a stock based solely on the potential trends of the past price. Numerous patterns are employed. Alongside the patterns, statistical techniques are utilized such as the Simple Moving Average (SMA), Relatively Strength Index (RSI), etc.

Technical analysis is very important to know stock market trend, breath, strength of trend. Technical indicators reflect the inside mirror image of stock market stocks. Using some technical indicators investors can understand the vital secrets inside the market. Exit and entry secrets are significant for successful investing. The purpose of an entry technique is to get a stock when it is getting ready to enter an uptrend and to avoid those stocks that are in a downtrend or a long basing pattern. The point of an exit methodology is to sell a stock at the time when it seems the uptrend is over or at least before a downtrend has progressed extraordinarily far. Stock trading is a process totally based on time so when predicting the stock market related data timing type stock trading indicators act a major role. Timing type stock trading indicators can mainly divide into two main parts. They are leading indicators and lagging indicators.

As the name implies, leading indicator [4] are better at predicting possible future trend. It precedes price movements and is often used to generate buy and sell signals. Leading Indicators are good at telling whether a stock's price has gone too high up or too far down, and whether there is a slowdown in price movement. If the stock's price has gone too high up, we say that the stock is now overbought. If the price has gone too far down, we say it is oversold. In either case, the leading indicators will show that the stock will not remain overbought or oversold for long. A pullback is imminent. Leading indicators are unable to extract the exact information of the trend pattern of the stocks. It helps investors to predict the behavior of the stock trading process so these types of indicators only provide the warning signals. But it is unable to confirm it really happen or not. So, the common problem of only using this type of technical indicators for the technical analysis process is that in some cases a trade could be opened too early and the signal could be ignored (no reversal).

Lagging Indicators are indicators which follow the stock's price pattern; hence the name "Lagging" was introduced to indicate this type of Indicators. Lagging indicators deals with the past stock data, they are good in showing whether a trend is developing, or whether at stock is in a trading range (i.e. trading sideways). Lagging indicators are not good at predicting future but they can show what trends have developed until the current point. These studies are more reliable than the leading technical indicators. However, they have other problem: in many cases a trade could be opened and closed when it is too late because it does not provide any warning signals at the beginning so investors may react at the trend already in reversal movement.

So, using these two types of indicators individually does not lead investors to a better trading decision. But combination of lagging and leading indicators allows substantially increase profitability of a trading system. Proposed system used three efficient timely type indicators. They are one leading indicator called Relatively Strength Index and two lagging indicators called Bollinger Bands and Moving Averages. The leading indicators could be used to generate a signal and alert a trader about possible reversal. Then lagging technical indicator would be used to confirm this 
reversal and open or close a trade. So proposed system was able to accurately guide the investors for their transaction decision.

\section{Moving Average}

Moving average [5] smoothers the closing price data to form a trend following indicator. The two most popular moving averages are the Simple Moving Average (SMA) and Exponential Moving Average (EMA). Moving averages help to smooth price action and filter out the noise. They also form the building blocks for many other technical indicators and overlays, such as Bollinger Band. Moving average basically is used to identify the direction of the trend or define potential support or resistance level. As considering both MAs SMA became the preferred method for tracking market prices because they are quick to calculate and easy to understand. Also, SMA would work well when looking at longer time frames, as it could give an idea of the overall trend. By considering these facts our technical analysis process we use the Simple Moving Average.

A Simple Moving Average is formed by computing the average price of stocks over a specific period of time. Most moving averages based on closing price. A 20-day SMA is the sum of last 20 day closing prices and divided by 20 .

\section{Bollinger Band (BB)}

Bollinger Bands [6] are a technical trading tool created by John Bollinger in the early 1980s. Those are volatility bands placed above and below a simple moving average. Volatility is based on the standard deviation, which changes a volatility increase and decreases. The bands automatically widen when volatility increases and narrow when volatility decreases. This dynamic nature of Bollinger Bands also means they can be used on different securities with the standard settings.

The purpose of Bollinger Bands is to provide a relative definition of high and low. By definition prices are high at the upper band and low at the lower band. This definition can aid in rigorous pattern recognition and is useful in comparing price action to the action of indicators to arrive at systematic trading decisions. Bollinger Bandwidth and Bollinger Percent [7] are used in technical analysis for the same purpose of volatility evaluation. Bollinger Bands allows you to visually evaluate volatility, whereas Bollinger Bandwidth represents volatility as a numeric value obtained by subtracting the lower band from the upper band, and the Bollinger Percent locates the current close price in relation to the bands by applying the stochastic formula:

$$
\begin{aligned}
& \text { Percent B }=((\text { Close }- \\
& \text { Bollinger Lower Band }) / \\
& (\text { Bollinger Upper Band }- \\
& \text { Bollinger Lower Band })) * 100
\end{aligned}
$$

Very often volatility is associated with investors' panic. Investors believe that a high value of volatility translates into a greater degree of market uncertainty, while a low value of volatility is consistent with greater stability. For instance, the volatility values greater than 30 are associated with a large amount of volatility as a result of investor fear. On the other hand, values below 20 correspond to less stressful times in the markets.

Bollinger Band Squeezes shown in fig 1 occur [8] when volatility falls to a very low level. This evidenced represent by narrowing upper and lower bands. The upper and lower bands are based on the standard deviation, which is a measure of volatility. 
Therefore, volatility agrees as the bands narrow. The bands narrow as price flattens or moves within a relatively narrow range. The theory is that periods of low volatility are followed by periods of high volatility. Relatively narrow band width or the Squeeze can predict a significant advance or decline. After a Squeeze, a price surge and subsequent band break signal the start of a new move. A new advance starts with a Squeeze and subsequent break above the upper band. A new decline starts with a Squeeze and

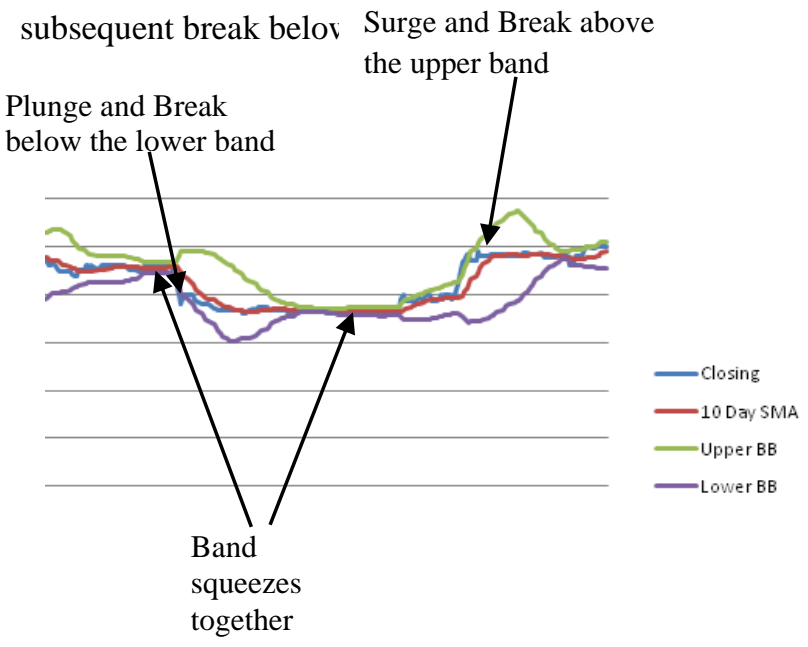

Fig 1 - Bollinger Band squeezes

Head and Fake pattern in the Bollinger Band is a rapid break of the opposite band. There is a big run up through the upper band then narrowing the bands (squeezes) and suddenly move to the opposite direction and touches the lower band. In the case of a downside move, traders could have been overly exuberant in taking profits after such a big run up to the high.

Bollinger bounce shown in Fig 2 occur when a stock's price will "bounce" off the upper or lower
Bollinger Bands and then return back towards the middle of the Bollinger band.

Price bounces back

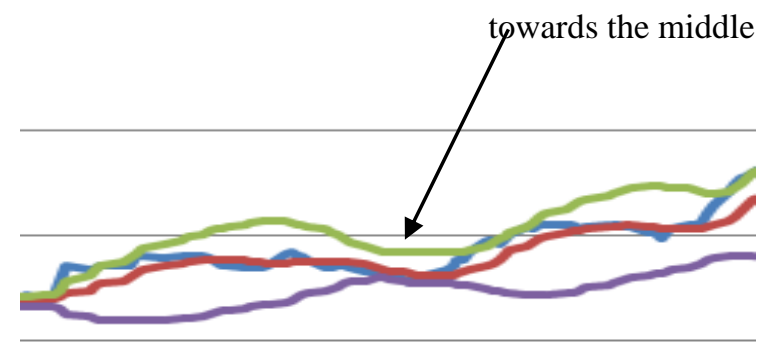

Fig 2-Classic Bollinger bounce

The fig 2 shows the classic Bollinger bounce. The reason these bounces occur is because Bollinger bands act like dynamic support and resistance levels. The longer the time frame you are in, the stronger these bands tend to be. Many traders have developed systems that thrive on these bounces and this strategy is best used when the market is ranging and there is no clear trend.

\section{Relatively Strength Index (RSI)}

Developed J. Welles Wilder, the Relative Strength Index (RSI) is a momentum oscillator [9] that measures the speed and change of price movements. The RSI compares the magnitude of stock's recent gains to the magnitude of its recent losses and turns that information into a number that range from 0 to 100. It takes a single parameter, the number of time periods to use in the calculation. Wilder recommends using 14 days.

Generally, it considers a stock as Overbought when the demand is too high that it causes the price to reach extremely high levels and effect courses an oscillator to reach the upper limits. A stock is said to be oversold 
when its price has become much lower than its normal value, making the oscillator reach the lower limit.

Failure Swings or momentum failure [10] is shown in Fig 3 occurs when RSI fail to swing back into its previous oversold or overbought conditions especially if it has reached those conditions more than once in a given period. This represents that the momentum has weakened and reversal is most likely to occur.

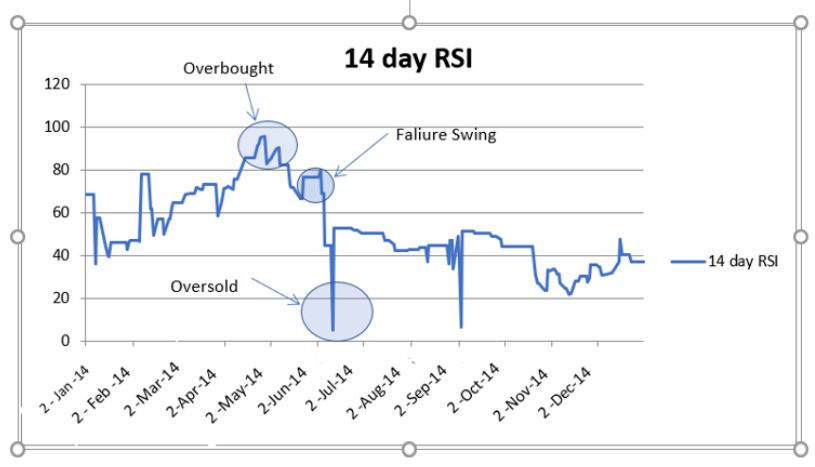

Figure 3 - Failure Swing of RSI

Divergence occurs when price of a stock and the value of an indicator move in opposite direction. Traders observe the market for any occurrence of divergence, which indicates changes in trend direction. Bearish divergence is when a stock price forms higher high while RSI forms lower highs and Bullish divergence is when a stock price forms lower lows while RSI forms higher lows.

When the price of an asset is unable to fall below a certain price for a period of time, that level is called Support. Conversely, when the price fails to climb higher than a certain level for a period of time, that level is called Resistance.

This system was tested their approach with ten most stable companies function within five sectors of the Colombo Stock Exchange (CSE). For each day ordinary or voting stock's (N) Highest, Lowest and
Closing value were obtained. Further, the share volumes were also obtained. Missing values were filled using the previous day stock prices. The data used for this study were obtained from the data library CD owned by CSE publication. Data were collected in all 2153 trading days from $1^{\text {st }}$ of January 2008 to $31^{\text {st }}$ of December 2017.

Since the birth of the stock market there exists a philosophical difference in how investors use the market to make money. Investors can be divided into three types called short term, medium term and long term. Short-term investors who see value in riding a given stock on its way up, and then selling at a nice profit. Normally short term refers to 1-3 months. The other investors called medium and long-term investors do not look at short-term ups and downs of a given stock, rather at the medium or long-haul view of several months or years. Medium term refers to 3 months -2 years. Long term refers to $2-20$ years. Proposed system was completely base technical analysis method to forecast the stock market so it mainly targets the medium and long-term investors. Technical analysis prediction method is analyzing the historical data and looks at the market trends so it's got some time periods to provide the result therefore this system mostly suited for the medium and long-term investors.

\section{Results}

This system discovered three prediction rules based on the three indicators as discussed before. First predict the trend using the leading indicator called RSI and then confirms it using two lagging indicators called BB and SMA.

Time frame: daily

Indicators: 
14 days Relatively Strength Index (14 RSI, according to the data analysis support and resistant level for the system respectively 70 and 30 used)

20 days Bollinger Band (20 days SMA considered as a Middle band, 20 days standard deviation with multiplier 2 is used to calculate lower and Upper Bands)

5 days Simple Moving Average (5 SMA)

20 days Simple Moving Average (20 SMA)

Fig 4 and Fig 5 confirms the Rule1 in the system. Charts are described by the CEYLON TOBACCO COMPANY PLC's (CTC) stock values variation within the Jul 2014 to Nov 2014 months. CTC company is belonging to the Beverage Food \& Tobacco sector.

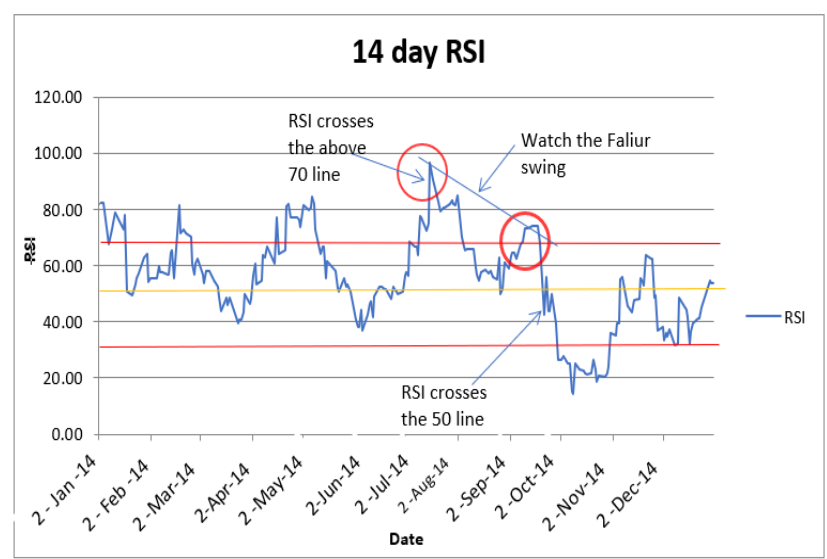

Figure 4 -14-day RSI chart for CTC (Year 2014)

After automatically generating the above charts (Fig 4) by the system investors can watch them carefully and forecast the market behavior. When watching the RSI chart, first investor can detect that the RSI crosses above the overbought limit (i.e. RSI above 70) at the middle of July. Further watching investor can detect the failure swing in between middle of Jul to middle of Sep. This behavior warns the investor there is a stock price decline within the near future.

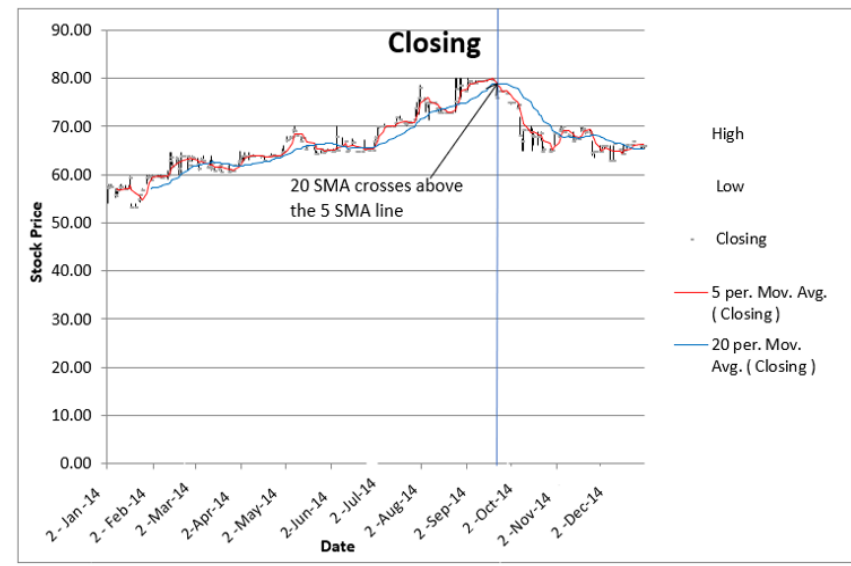

Fig 5 Stock chart with trend lines for CTC (Yea2014)

So, keep the above analytical knowledge in his mind he watches Fig 4 and Fig 5 both graphs to confirm that. As the confirmation he can see on $26^{\text {th }}$ of Sep 2014 RSI crosses the neutral limit (i.e. RSI is 50) simultaneously 20 SMA crosses above the 5 SMA. Sell trade rule 1 is satisfied so investor can enter to the market and sell their stocks because investor can predict price is further reducing in the near future.

Fig 6, Fig 7 and Fig 8 confirm the Rule 2 in the system. Charts are described by the behaviors of the JOHN KEELLS HOLDINGS PLC's (JKH) stock values within the Jun 2013 to Sep 2013 months. JKH company is belongs to the Diversified Holding sector. 
After automatically generating the below charts by the system investor can analyzed them according to the rules defined by the system.

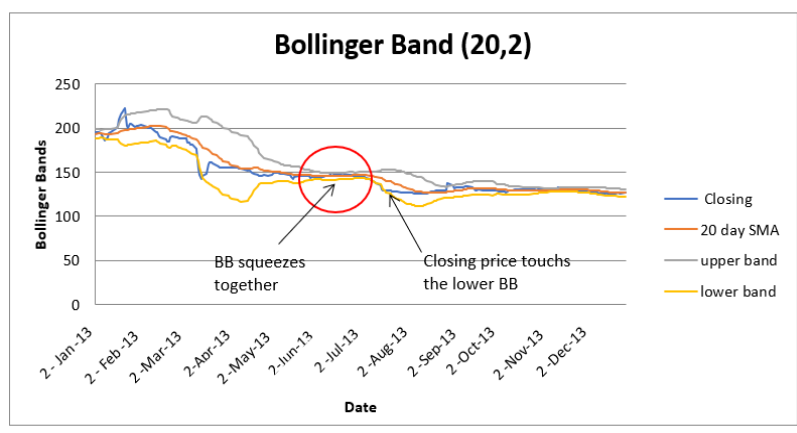

Fig.6 -Bollinger Band (20, 2) chart for JKH (Year 2013)

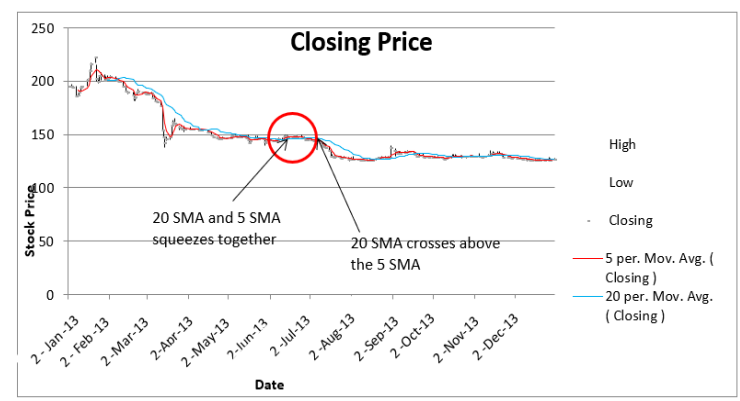

Fig 7-Stock chart for JKH (Year 2013)

Investors, first watch the behavior of the Bollinger Band $(20,2)$ and Closing price with 5 and 20 SMA trend lines graphs (Fig 5 and Fig 6). When analyzing those charts Investors can watch within the range of end of May to end of July BB and SMA are squeezes together. This behavior warns the investor within the near future he can face subsequent decline or advance of the stock price. He can wait until the closing price

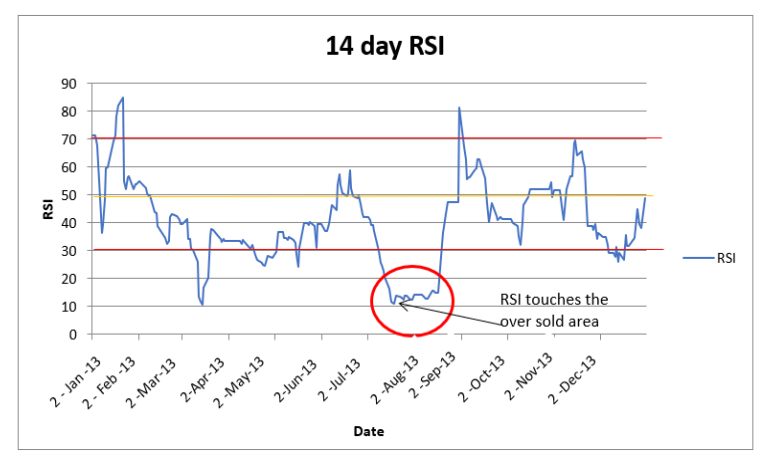

This publication is licensed under Creative Commons Attribution CC BY. http://dx.doi.org/10.29322/IJSRP.11.01.2021.p10993 touches the lower BB and 20 SMA crosses above the 5 SMA.

Fig 8 - 14-day RSI chart for JKH (Year 2013) Also, RSI touches the oversold area. According to the Fig 8 all of these behaviors reach on $11^{\text {th }}$ July 2013 confirm the decline.

Charts shown in Fig 9 and Fig 10 confirm the Rule 3 in the system. Charts are described by the SRI LANKA TELECOM PLC's (SLTL) stock price variation within the 2004 Apr to 2004 May and 2004 Oct to 2004 Nov. SLTL Company is belong to the Telecommunication sector.

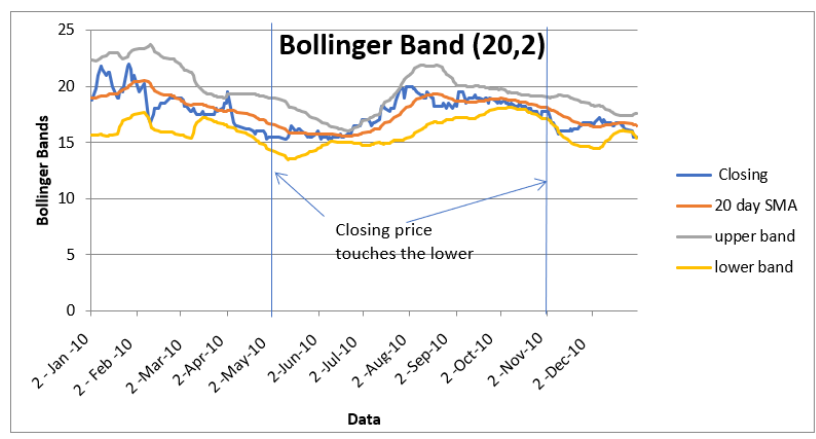

Fig 9- Bollinger Band (20,2) Chart for SLTL (Year 2010)

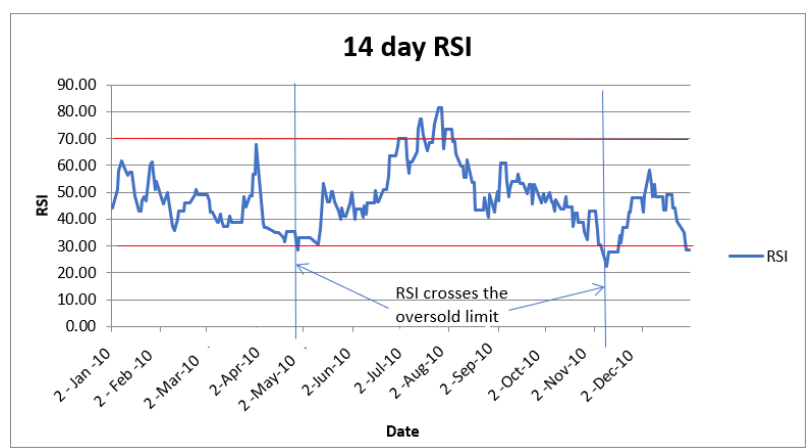

Fig 10- 14 day RSI Chart for SLTL (Year 2010)

After automatically generating the above charts by the system investors can analyzed them according to the rules define in the system. While watching the above charts investor can detect $28^{\text {th }}$ April 2010 and $8^{\text {th }}$ Nov 2010 closing price touches the lower BB and RSI 
crosses the oversold limit. So according to the buy trade rule3 define by the system investor can predict there is a price reversal within the next few days so investor can enter to the market and buy the stock at lower prices.

\section{Conclusion}

Final conclusion is that investors can win their trading process when they effectively using this system. Proposed system is totally base on the three rules. First two rules define on the system are mostly helpful for the medium-term investors and final rule helpful for the short-term investors.

\section{References}

[1] StatSoft. (2019, Mar). Data Mining Techniques. [Online]. Available: http://www.statsoft.com/textbook/data-miningtechniques/\#pdm

[2] (2019, Aug.). Stock Market Prediction. [Online]. Available:

http://www.learnartificialneuralnetworks.com/stockm arketprediction.html

[4] (2019, Sep.). Technical Indicators. [Online].

Available:

http://www.option-trading-

guide.com/technicalindicators.html

[5] (2019, Sep.). Moving Averages-Simple and

Exponential. [Online]. Available:

http://stockcharts.com/school/doku.php?id=chart_sch

ool:technical_indicators:moving_averages

[6] (2019, Sep.). Bollinger Band.[Online]. Available:

http://stockcharts.com/school/doku.php?id=chart_sch

ool:technical_indicators:bollinger_bands

[7] (2019,Sep.). Technical Analysis, Studies,

Indicators: Bollinger Bands. [Online]. Available:

http://www.marketvolume.com/technicalanalysis/boll inger_bands.asp
[8] (2019, Sep.). Bollinger Bands Introductions.

[Online]. Available: http://www.bollingerbands.com/ [9] (2019, Sep.). Relatively Strength Index. [Online]. Available:

http://stockcharts.com/school/doku.php?id=chart_sch ool:technical_indicators:relative_strength_index_rsi [10] (2019, Sep.). Relatively Strength Index.

[Online]. Available:

http://www.surefiretradingchallenge.com/blog/relativ e-strength-index-rsi

\section{Author}

A.V.L Chandima, Master of Computer Science, University of Colombo. Sri Lanka Institute of Advanced Technological Education (Department of IT, Advanced Technological Institute -Gampaha), lalanee@Sliate.ac.lk 